\title{
Incorporating EFFECTs into Content-Rich Courses: A Journey
}

Dr. Rebecca Bourn, University of Wisconsin - Milwaukee

Dr. Bourn is the Calculus Coordinator at the University of Wisconsin-Milwaukee. She earned her Ph.D. in Applied Mathematics from the University of Virginia School of Engineering and Applied Science.

Dr. Sarah Baxter, University of St. Thomas

Dr. Baxter is a Professor of Mechanical Engineering in the School of Engineering at the University of St, Thomas in St. Paul, MN. She received her PhD in Applied Mathematics from the University of Virginia School of Engineering and Applied Science. 


\section{Incorporating EFFECTs into Content-Rich Courses: A Journey}

Many foundation courses in mathematics are considered "content-rich," meaning they must cover a significant amount of material to introduce tools and methods that will be used in subsequent courses. These courses are tightly scheduled surveys of multiple topics; all of which are considered "essential". The presumption is that students will build skills and develop expertise in these foundation topics and be able to recall and use them when required, at a later date, in a future course. Anecdotal evidence suggests that frequently students will do well in the initial course but retain little familiarity with the content in later courses, when context, application, and sometimes even notation can be quite different. This is often true for engineering students with respect to the Calculus sequence.

In courses such as Calculus, concepts and solution methods are typically presented within a mathematical context. While some students can recognize the underlying structure and the mathematical construction, others have trouble identifying patterns or parallel thought structures, which makes it difficult for them to generalize the concept to a range of problem types. For example, students in an Introduction to Mathematical Statistics course were reported to claim they do not know how to integrate a probability distribution over a region. The pre-requisite for the course is Multivariable Calculus, which most certainly covers this topic, but the problem "feels" different to students in the later course because the notation and setting have changed and the purpose is specific to statistics rather than the more abstract concepts of the area of a twodimensional region and anti-derivatives.

\section{Previous Work}

In recent years the authors have been exploring ways to reframe course assignments to provide a greater variety of application and visualization avenues to enhance critical thinking and prompt student reflection. The objective is to provide multiple levels of connections that promote students' cognitive retention. Preliminary work ${ }^{1}$ presented a methodology for using large scale, Fermi-type estimation problems to try to encourage students to "see/recognize" geometry in their surroundings and in problem solving, in order to ground the concepts and help them develop a sense the reasonableness in their answers. Estimating forced students to ignore their "tools" but they were still able to provide a quality solution to the large-scale problem. The exercises cast math as a useful tool instead of an abstract language. These projects were well-received by students; surveys suggested that they felt like they were doing something with the math. These projects were offered in classes that emphasized problem solving, but had more flexible content and learning objectives.

The next step was to identify ways to extend the underlying concept, by adding context into content rich foundation courses. One approach was presented by a faculty group at the University of South Carolina. With NSF support they developed a pedagogical framework to assist students in deepening their conceptual understanding of new and challenging ideas. This framework, known as EFFECTs (Environments for Fostering Effective Critical Thinking Skills), was originally aimed at introductory civil engineering students, but has been extended to upper division engineering classes as well. The format intertwines multiple learning modules and written journal entries into a multi-session exploration of a large-scale physical problem. Often the problem has multiple best designs rather than a unique solution. Critical thinking skills are 
enhanced through student reflection on the ideas, implications, or inherent challenges of developing a solution, prior to and along with, formal solution techniques. In their research they have demonstrated improvements in student engagement, technical skills, and deeper growth of understanding of core knowledge ${ }^{2}$.

The classic example of EFFECTS appears in the context of a geotechnical engineering course exploring the question of "How much soil will it take to rebuild the levees destroyed by Hurricane Katrina". Faculty had noticed that students would offer a "solution" but had no sense of its quality or accuracy. The instructors created a framework that began with the presentation of an imagination-capturing engineering scenario, followed by one or more modules of instruction or exploration on topics relevant to the solution of the problem such as the weight of soil, the effects of compaction, the efficiency of soil dams. Each module concluded with a mandatory journal-type entry in which students explain their understanding of what they learned and its relevance to the overall driving questions. They found that with this reflective writing component added to the assessments, students became better able to identify their own learning and find gaps in their knowledge. As a result, students have better retention of what they are learning and a deeper understanding of its importance. The original EFFECTs work included some formal study of assessment of improved cognition ${ }^{2}$. They found statistically significant improvement for students using the EFFECTs framework.

In the second stage of this work ${ }^{3}$, the authors began to explore ways to add reflective thinking and adapt the EFFECTs framework into the math classroom, Math-EFFECTs. The approach incorporated physical applications, geometry-based problems, and estimation techniques into solution processes to contribute to the "does this answer make sense" aspect of critical thinking. These Math-EFFECTs modules were proposed for courses that still had flexible content requirements that allowed for more free form, less time constrained exploration. Students reported more enjoyment in learning and that their feelings of creativity in using mathematics had increased significantly as they (1) completed realistically modeled problems and (2) were given the opportunity to make choices regarding approaches to problem solving. The response to the conference presentation on this work was positive, however the audience pointed out the difficulty of finding time in content rich classes for the more extended time required to implement an EFFECTs module.

\section{Current Work}

There is seldom time (or expertise) to redesign levees in a calculus class. However, the fundamental ideas embedded in the EFFECTs framework still seem relevant to mathematics. In the current work, the authors explore the feasibility of applying EFFECTs for mathematical concepts in content-driven courses.

Three components from the EFFECTs framework seem critical: (1) a visual, or tactile component, (2) a connection to something familiar, and (3) student reflection on how this addition to the lecture changed their perception and understanding of the concept.

An obvious way to meet the first two goals is an in-class demonstration. This provides a visual that corresponds to a math concept and could be added to a lecture with relatively short time commitment. If there is any physical manipulation involved in the demonstration, or measurements to be made, a demonstration would also offer a tactile component. In general, a 
demonstration can easily be based on objects with which students are familiar. Experiment based demonstrations, however, are not consistently a good match to math. Good ones take more time to present, and may be difficult to focus towards one curriculum-based set of concepts. In addition, while a demonstration may capture a student's attention, it does not provide a structure for increasing understanding by exploring complex problem solutions through reflections.

It is this third component to the EFFECTs approach (student reflection) that is key to the success of the framework. It is also the most challenging component to include in the context of mathematics, where there is little opportunity to contribute an individual opinion on the topic. However, it is important to the goal of increasing longer-term retention of information that students have an opportunity both to reflect on the mathematical technique and to offer conjecture on its future utility. In the following sections specific visual examples on two mathematical concepts are provided and beginning attempts to include a reflective component are discussed.

\section{Visual Examples}

Another option is the use of visual examples. These are in contrast to problem-solving examples that are worked by the instructor on the board or in a presentation. These could be actual demonstrations with an embedded measurement, for example, the connection between a derivative and the slope of stairs, or a ramp; or a simple video such as water filling a tank from a hose to motivate related rates ${ }^{4}$. These visual examples should serve to anchor, motivate and/or provoke questions about the concept. The emphasis is on the requirement that the example be something physically tangible and familiar to the students, not taken from, or viewed solely on the pages of a book.

In short, the goal of a visual example is to retain key elements of the EFFECTs framework but minimize added instruction time. In particular, the two topics of solids of revolution (Calculus II) and cross-products (Calculus III) are presented. The basic component of each module is a strong visual demonstration that is connected to the underlying mathematics. These visual examples highlight the fact that they can be very simple and inexpensive. While the goal is to anchor, motivate and/or provoke questions about the concept, by interjecting these short examples into a lecture format, an element of active learning is also added to the class.

\section{Cross-Product}

In the case of the Cross Product, the Math-EFFECTs visual example was presented in a Statics class. Although students admitted having had cross products presented in Calculus and Physics, they had difficulty remembering how to calculate a cross product, how it was different from a dot product (scalar product), and they were unable to determine if what they calculated made sense. The application, specific to this class, was that a cross product is used to calculate a moment. Moments describe the tendency of a force to try cause rotations (Vectors, forces and moments are denoted by bold face.) For students, the scalar, two-dimensional moment,

$$
|\mathbf{M}|=|\mathbf{F}| \cdot d
$$

magnitude of force, times distance of moment arm, made sense.; how the cross product, position vector, $\mathbf{r}$, time force vector, $\mathbf{F}, \mathbf{r} \times \mathbf{F}$, reflected this, didn't. As a result, students resisted using 
the cross-product even in cases where decomposing the problem into scalar products was difficult to do.

The demonstration required a block of wood, duct tape, and cord attached to one corner of the block. The instructor wrote out the calculated approach to solving $\mathbf{r} \times \mathbf{F}$

$$
\begin{aligned}
\mathbf{M} & =\left|\begin{array}{ccc}
i & j & k \\
r_{x} & r_{y} & r_{z} \\
F_{x} & F_{y} & F_{z}
\end{array}\right|=\mathbf{i}\left|\begin{array}{cc}
r_{y} & r_{z} \\
F_{y} & F_{z}
\end{array}\right|-\mathbf{j}\left|\begin{array}{cc}
r_{x} & r_{z} \\
F_{x} & F_{z}
\end{array}\right|+\mathbf{k}\left|\begin{array}{cc}
r_{x} & r_{y} \\
F_{x} & F_{y}
\end{array}\right| \\
& =\left(r_{y} F_{z}-r_{z} F_{y}\right) \mathbf{i}-\left(r_{x} F_{z}-r_{z} F_{x}\right) \mathbf{j}+\left(R_{x} F_{y}-r_{y} F_{x}\right) \mathbf{k}
\end{aligned}
$$

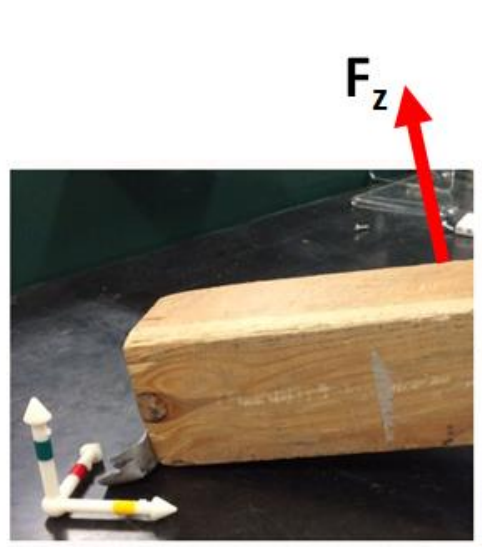

(a)

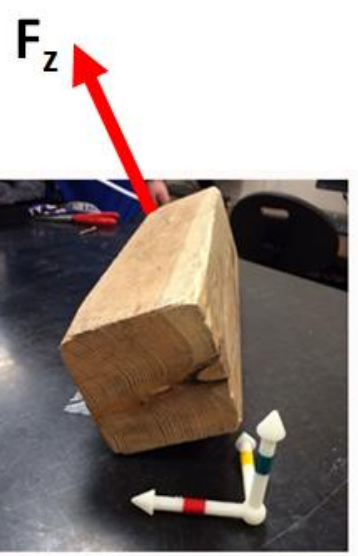

(b)

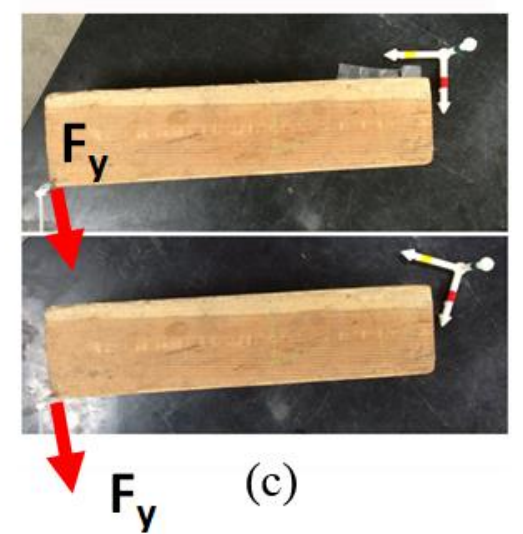

Figure 1: The z-component of a force can be show to contribute to a rotation about y (a) and $\mathrm{x}$ (b) axes. This corresponds to the presence of $F_{z}$ in the $M_{x}$ and $M_{y}$ terms of the formula in eqns. (3) and (4). Similarly, the component of the force in the $\mathrm{y}$-direction contributes to a rotation about the $\mathrm{z}$ axes (c) and $\mathrm{x}$ axis, (not shown), and can be matched to the presence of $F_{y}$ in the $M_{x}$ and $M_{z}$ components of the moment vector.

$$
=M_{x} \mathbf{i}+M_{y} \mathbf{j} M_{z} \mathbf{k}
$$

(The $x, y, z$, coordinate unit vectors are denoted, $\mathbf{i}, \mathbf{j}, \mathbf{k}$.) The block was then attached to a surface on one side with the tape (Figure 1), with the coordinate axes identified as lying along the directions of the sides of the block.

The cord was used to apply a force, pulling on the block in each of the coordinate directions, in turn. How the block rotated, around which axes, could be clearly connected to the components resulting from the three parts of the cross-product. Pulling in the $z$ direction would rotate the block about both the $x$ and $y$-axes. In eqns. (2), (3), the $z$ component of the force appears in both 
the $\mathrm{M}_{\mathrm{x}}$ and $\mathrm{M}_{\mathrm{y}}$ terms. Thus, a force in the $y$ direction will rotate the block about the $x$-axes and the $z$-axis, and a force in the $x$-direction, a moment about the $y$, and $z$.

In previous classes, the instructor had found that students were unwilling to use the crossproduct, instead trying to do the 2-dimensional visualization with the scalar calculation of equation (1). When the axes are not orthogonal, or there is a complicated three-dimensional representation, this is difficult. After making the connection of the cross-product math to the rotation of the block, more students appeared willing to use, and trust, the math. There were also able to provide an answer to the "does this make sense" question, i.e., "will this force cause a rotation about this axis?"

\section{Volumes of Solids of Revolution}

Solids of Revolution is a standard calculus topic that combines multiple skills including sketching, geometrical analysis, and integration. In the Math-EFFECTs context, the related topics of area between curves and volumes of solids are used in the framework of a driving question that asks, "How much material will it take to make this plastic game piece?" This sets a tangible purpose for why the topic is being approached and a visualization of what a solid of revolution looks like in "real life."

The initial study on area between curves now has a different setting. It is no longer a disconnected effort of creating Riemann sums for arbitrary shapes, but rather an exercise in creating interesting object profiles using functions that are easy to draw and integrate. It also

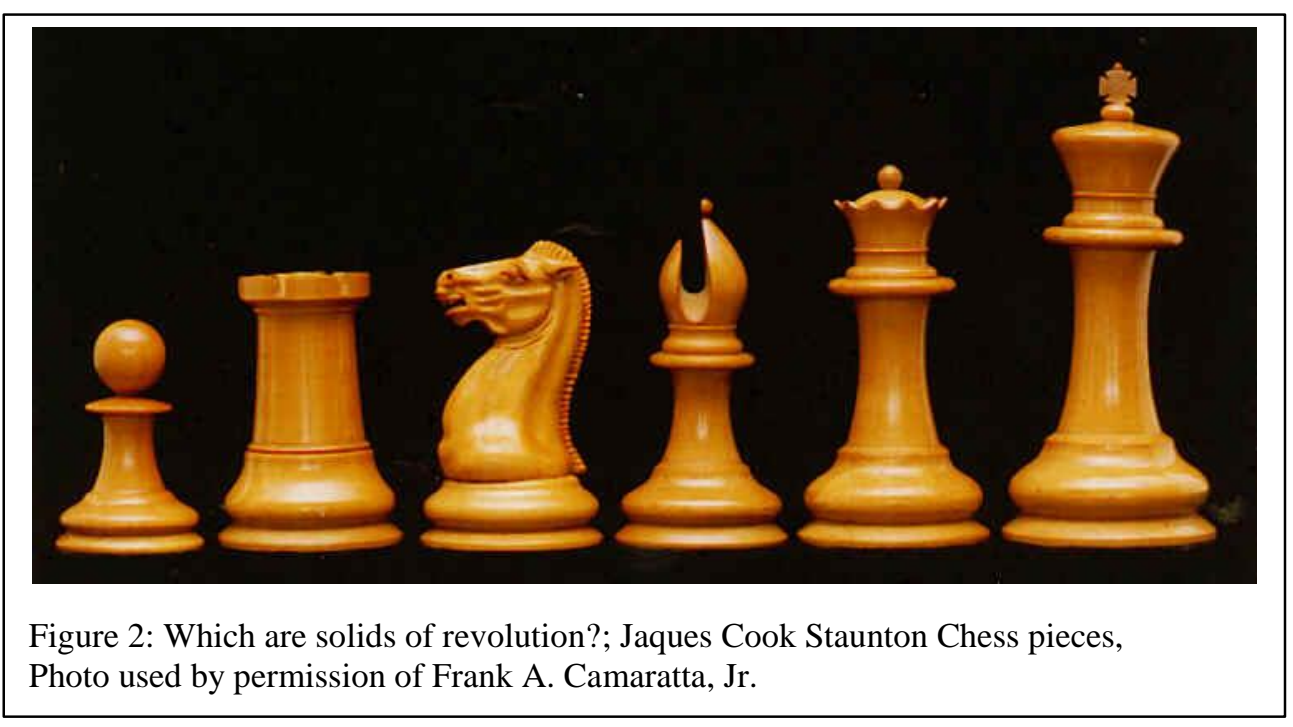

lends itself as the background for questioning the process and application of creating solids. Students are asked to reflect on the process of creating an object's profile, and what kinds of functions are well-suited for that purpose. Students are also asked to explain the changes or complications that arise when using a vertical rather than horizontal axis.

Instruction on calculating volumes using disks (washers) and shells can then proceed in the usual fashion, using a variety of problem solving examples that showcase both methods, present rotation about either axis, and use either $\mathrm{x}$ or $\mathrm{y}$ as the variable of integration. The capstone 
experience for these sections is a laboratory-type experience where students analyze a collection of actual physical objects. The collection of objects includes game pieces (of course), and variously shaped vases, candleholders, and even a snowman. One seasonal example is that of paper Christmas bells that are initially flat, but open into a three-dimensional shape using the exact motion that creates a solid by revolution about an axis.

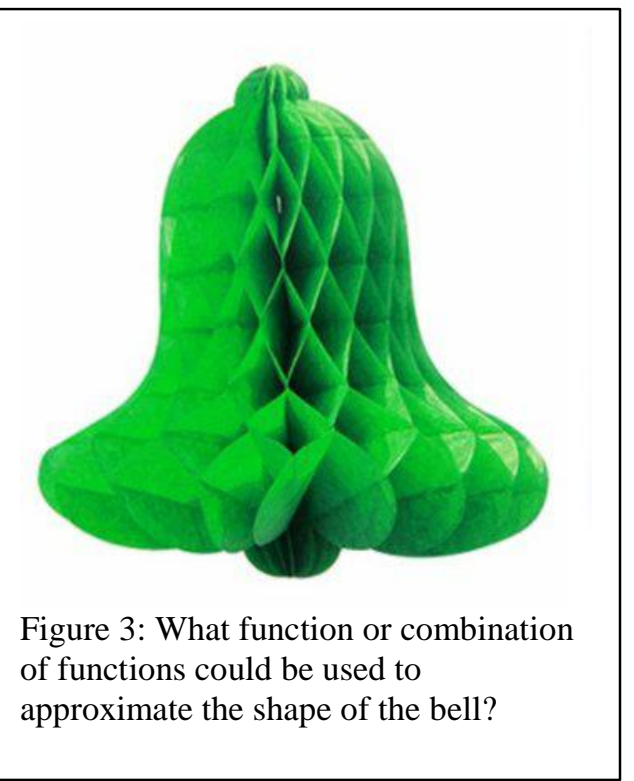

Students are guided through an analysis of their object by estimating a maximum volume, drawing the object's profile, estimating its shape with piecewise defined functions, and creating integrals and calculating a more precise volume estimate. Familiar functional approximations such as polynomials or sinusoidal curves can be used for the revolving functions. Finally, students are asked to compare the quality of their original volume estimate compared to predicted volume, and reflect on their design process and how easy (or difficult) it was to work with real objects.

\section{Reflection Exercises}

The most challenging part of adopting the EFFECTs approach for mathematics modules is to find a way to include an aspect of reflection. Visualization and demonstration are powerful tools, but a reflection

component can help make students more aware of how they learn and when the learning is based on internalizing the information, rather than memorizing. Because most of our students approach mathematics from the perspective of engineering, demonstrations have wide appeal, writing an essay does not. The approach that has been the easiest to implement so far has been short guided discussions, and greater transparency in explaining to the students why the visual examples were being included and what the instructors hoped they would help students achieve.

The authors started by trying to ask questions like "Does this help you understand this type of problem" at appropriate places. This worked to initiate discussion and pointed out the instructional goals. The potential exists to ask more sophisticated questions, for example, "when would you use the scalar formulation rather than the cross-product formulation" or "what features of an object point you towards using shells versus washers when calculating volumes?"

In presenting the cross-product module, the initial demonstration was with a thinner board. Students smiled and politely nodded but did not seem inspired. In consultation with the Department lab manager, who identifies with visual understanding, the thicker block of wood was substituted. When asked which worked better and why, students responded that the thicker block worked better because the moment arms were big enough to see, and the rotation easier to imagine. After the demonstration, students were asked to work book problems, and many came up and rotated the block around axes to mimic the problem that they were working on. Near the end of the class, students were asked to respond on the usefulness of the cross-product/moment demonstration. The majority reported that seeing why the formula made sense made them more willing to trust the math in more complex problems. They also saw the block example as a way to determine if the answer provided by the cross-product made sense. 
In the Solids of Revolution module student engagement in the design activity was incredibly strong. They seem very interested in the project and several students brought in their own objects. However, they seemed to struggle enormously in identifying reasonable approximate curves for the object profile. The benefit of this struggle was a good discussion about elementary functions. Also, the apparent gap in comprehension has led to a better presentation of the 'area between curves' material covered earlier; emphasizing it as part of a design process rather than a strictly mathematical exercise. Eventually it would be interesting to work with 3-d printing of the modeled objects so that students can see how closely their mathematical models represented their physical objects.

\section{Assessment}

The major assessable component of each of these modules is in student involvement in the demonstrations. Objects to handle and examine are simple approaches to adding an active learning component to the class. The research has clearly shown that active learning, even as a relatively small component of the class, can have a number of positive outcomes ${ }^{5}$. The more subtle objectives, improved critical thinking skills, more fundamental retention of math skills, and potentially better visualization skills are difficult to assess, especially in the short term ${ }^{6}$.

A long-term goal of this work is to develop better metrics for more specific outcomes related to mathematics, and do more formal assessments. Several possible metrics are concept exams and more consistent student surveys.

\section{Future Work}

The main focus of future work will be to develop a library of simple visual examples, specifically for the mathematics classroom, and to experiment with approaches to include student reflection on their understanding as well as on their own learning styles. These modules easily fall into the category of an active learning exercise, but additional assessment metrics, perhaps with more focus on the degree to which students recognize this type of approach as valid, are needed.

\section{References}

[1] Bourn, R., and Baxter, S. C. (2013), Developing Mathematical Intuition by Building Estimation Skills, Paper presented at 2013 ASEE Annual Conference, Atlanta, Georgia. https://peer.asee.org/19419

[2] Pierce, Charles E., Gassman, Sarah L. and Huffman, Jeffrey T. (2013) "Environments for fostering effective critical thinking in geotechnical engineering education (Geo-EFFECTs)" European Journal of Engineering Education, 38:3, 281-299,

[3] Bourn, R., and Baxter, S. C. (2014), Creating Environments for Fostering Effective Critical Thinking in Mathematics Education (Math-EFFECTs) Paper presented at 2014 ASEE Annual Conference, Indianapolis, Indiana. https://peer.asee.org/20223

[4] Berger, Warren, A More Beautiful Question: The Power of Inquiry to Spark Breakthrough Ideas, Bloomsbury USA, NY 2014.

[5] Freeman, S., Eddy, S.L., McDonough, M., Smith, M.K., Okoroafor, N. Jordt, H and Wenderroth, M.P. “Active learning increases student performance in science, engineering and mathematics" PNAS, 111(23), 8410-8415

[6] Prince, M. "Does Active Learning Work? A Review of the Research" J. Engr. Education, 93(3) 223-231, 2004 